\title{
VARIA
}

\section{LA PRAGMATIQUE LUDIQUE DU TÉLÉROMAN : LE CAS "DALLAS"}

\author{
Philippe Sohet ${ }^{1}$
}

\section{Dallas : du succès au phénomène de société}

Le 21 novembre 1980 entre 80 et 110 millions de téléspectateurs américains étaient rivés devant leur écran de télévision pour suivre un épisode de Dallas (Qui a tué J.R. ?). Une telle audience représentait plus de $34.5 \%$ de la population et $80 \%$ des téléspectateurs potentiels : le plus haut taux d'écoute pour une fiction de prime-time ${ }^{2}$. Si l'indice le plus évident du succès d'une série est bien la mesure de son audience, Dallas, avec son écoute nationale de croisière d'environ 44 millions $^{3}$ de téléspectateurs fait figure de série mythique. L'étendue de ce succès ne s'est d'ailleurs pas restreinte au niveau

1 Professeur au Département des communications de l'Université du Québec à Montréal (UQAM).

2 Et qui ne semble d'ailleurs avoir été dépassée qu'une seule fois par la suite lors de la finale de Mash.

3 Si Stolz (1984) parle de 28 millions, Time (1980) avance le chiffre de 40 millions, et Newsweek (1980) 44 millions, données généralement admises dans le milieu. Dallas possédait ainsi l'audience la plus forte dans son genre.

Recherches en communication, $\mathrm{n}^{\circ} 10,(1998)$. 
national : avec ses 350 millions de spectateurs dans près de $97^{1}$ pays différents, Dallas afficha également la diffusion la plus large dans son genre. Une telle audience apparaît d'autant plus significative qu'elle a pu s'établir sur 14 saisons et environ 357 épisodes : une durée tout-àfait exceptionnelle ${ }^{2}$ pour une telle série. L'ampleur de l'audience n'est évidemment pas sans incidence sur les retombées économiques et, sous cet aspect également, Dallas semble offrir la panoplie des symptômes du succès : réputée la plus chère à produire ${ }^{3}$, s'autorisant des cachets extravagants ${ }^{4}$, cette série engendrera néanmoins des revenus publicitaires fabuleux ${ }^{5}$.

Dans une perspective plus proche de notre propos, il est également possible de jauger le succès d'une production-média aux réactions qu'elle aura suscitées dans l'univers des industries culturelles. A ce titre, Dallas fait figure de cas d'espèce puisqu'à sa suite on aura vu se déployer l'éventail complet des stratégies de positionnement industriel face à un succès. Stratégies de "clonage" pour les productions qui ne se cachent pas d'essayer de reproduire certains des éléments cardinaux de la série-vedette : à Dallas qui fait les beaux jours du réseau CBS, on opposera rapidement Dynasty (et sa suite The Colby) chez $\mathrm{ABC}$, ainsi que Flamingo Road à NBC. On parlera encore de Texas comme de la "day-time version of Dallas"' et même, chez CBS, de l'éphémère "The secrets of Midland Heights"7. Stratégie de

1 Les estimations évoluent graduellement selon la date de publication : en 1980, Time avance le chiffre de 57 pays, Cornu (1984): 60 pays, Bianchi (1984): 80 et l'émission Télé-dollars (1986): 97.

2 En fait la plus longue à ce jour pour un soap de prime-time hebdomadaire. On ne peut donc les comparer avec la longévité des séries qu'on appelle des anthologies (comme Walt Disney présente) ou des westerns (à épisodes autonomes) qui ont pu durer parfois 20 saisons (Gunsmoke) pas plus qu'avec le nombre d'épisodes de certains soaps quotidiens (le Coronation street britannique aurait approché les 2000 épisodes de trente minutes).

3 Ici également on assiste à une courbe exponentielle : 420.000 U\$ en 79-80 selon Bianchi (1984), 700.000 U\$ en 1983 selon Cornu (1983) et $1.960 .000 \$$ canadiens en 1986 d'après Télé-dollars (1986).

4 Durant cette période, l'acteur principal, Larry Hagman a vu son contrat passer de $7.500 \$$ par épisode à $140.000 \$$ plus un $\%$ des recettes! (selon The Gazette, 1986).

5 Les trente secondes de publicité sont estimées à 210.000 dollars par Télé-dollars alors que Le Figaro (1982) avance le chiffre de 250.000.

6 H. NEWCOMB, "Texas : a giant state of mind", Channels, vol. $1, \mathrm{n}^{\circ} 1$, avril-mai 1981, repris H. NEWCOMB, Television, the Critical View, 4e ed., Oxford University Press, 1987, pp. 419-432.

7 "Dallas-inspired soap-opera (with) an emphasis on the local teenage population" (The complete directory to prime time network, New York, Ballantine books, 1992, p. 791). 
"marcottage" en quelque sorte lorsque CBS entreprend la production de Knots Landing (Les héritiers du rêve). Cette série, centrée autour du personnage de Gary Ewing, autonomise un des frères de la célèbre famille de Dallas et se permettra de temps en temps des interférences d'une série à l'autre. Pour poursuivre dans la métaphore horticole on peut parler de stratégie de parasitage pour les inévitables réactions satiriques et parodiques. A côté des occasionnelles caricatures ${ }^{1}$, Malice (Salad Fork Ranch) ${ }^{2}$ se présentait comme une véritable série radiophonique. Et une telle énumération ne pourrait être complète sans mentionner les innombrables manifestations des stratégies d'essaimage : l'extension du succès de Dallas sur d'autres sphères. Il n'aura guère fallu de temps pour voir paraître plusieurs romans sur le sujet, des photos-romans, des récits en bandes dessinées et un projet de film, sans parler des "Compagnion books" et autres incontournables produits dérivés.

Ce qui précède suffit à inscrire Dallas parmi les grands succès des industries culturelles. Mais, outre ces indices classiques, Dallas aura provoqué d'autres types de réactions dont la nature nous pousse à croire qu'au-delà du simple succès commercial, Dallas peut être appréhendé comme un véritable phénomène de société.

Ainsi, jamais série télévisée n'aura-t-elle suscitée autant d'études académiques. Elihu Katz n'hésite d'ailleurs pas à en parler comme "the most studied of all television texts". Une compilation bibliographique, rapide et non exhaustive, permet déjà de relever quatre monographies universitaires exclusivement consacrées au sujet, une quarantaine de recherches (articles ou chapitres) directement concernées par le sujet (et presqu' autant d'autres qui y réfèrent avec quelqu'ampleur), ainsi que trois documents audio-visuels ${ }^{3}$.

Ce qui nous semble plus déterminant encore pour désigner un phénomène de société est ce que nous nommerions l'effet de contamination: la façon dont un phénomène culturel -Dallas- joue sur d'autres sphères que sa sphère d'origine (la culture médiatique), la manière dont sa valeur symbolique sera détournée pour désigner d'autres domaines et devient, par le fait même, un point de repère

1 Ne fut-ce que dans le célèbre magazine satirique $\operatorname{Mad}\left(\mathrm{n}^{\circ} 223\right.$, juin 1981).

2 Newsweek (1980). Volonté parodique que souligne déjà l'homologie phonétique des titres Dallas/Southfork Ranch et Malice/ Salad Fork Ranch .

3 De concert avec la désormais classique recherche de Katz (1984), on y retrouve des écrits sur la perception de Dallas se référant à des publics de pas moins de 16 contrées différentes. Voir annexe 2. 
social, une balise dans l'imaginaire. Ce qu'en d'autres termes on nommerait l'entrée de Dallas dans le travail intertextuel.

Les anecdotes qui jalonnent la diffusion de Dallas sont innombrables et de natures très variées, s'étendant des détails de la vie quotidienne ${ }^{1}$ à des dimensions sociales plus symboliques. Pour ne s'en tenir qu'au seul champ du politique, il est déjà frappant de voir les multiples occurrences du phénomène Dallas : aux États-Unis, en Israël, en Afrique du Sud des institutions traditionnelles et gouvernementales ont déplacé des activités pour préserver les heures de diffusion et, par ailleurs, le principal slogan que proclamaient les macarons distribués à la convention républicaine sous Reagan n'était autre que "A democrat shot J.R.". Là encore, l'impact de Dallas ne s'est pas restreint à la seule sphère domestique et rapidement la rumeur a voulu que Dallas soit un cadeau des USA à l'Algérie en remerciement de son intervention durant la crise des otages en Iran. A l'inverse, son retrait de diffusion aurait fait partie des conditions de l'ultimatum des fondamentalistes musulmans en Turquie. Toujours sur le registre diplomatique, on ne peut oublier le duel J.L. contre J.R. : la célèbre sortie du ministre français Jack Lang et la non moins percutante riposte du Wall Street Journal' ${ }^{2}$. Dans l'escarmouche Dallas acquérait le statut de symbole même de la culture "made in USA". On ne pourrait sans doute mieux mesurer la dimension symbolique acquise par la série qu'en se référant au glissement sémantique opéré par le vocable de son titre dans l'imaginaire populaire. Car qu'est-ce que "Dallas" dorénavant? Pour les critiques comme les spectateurs: Dallas remplace Dallas ; la fiction (télésérie) a devancé la réalité de la tragédie historique. Dans l'imaginaire, la dynastie des Ewing côtoie celle des Kennedy : les frères John (R) et Bobby Ewing ont pris la place de John (F) et Bobby Kennedy. Les attentats contre les frères Ewing ajoutant à l'homologie des prénoms ${ }^{3}$. La tentative d'assassinat

1 Par exemple, au Danemark, il avait été signalé comment la consommation d'eau et l'encombrement des lignes téléphoniques devenaient alarmants... aux moments des poses publicitaires du feuilleton.

2 "Dallas est le symbole de l'impérialisme culturel américain" dénonçait Lang au colloque "Culture et développement" organisé à la Sorbonne en février 1983 . A quoi le quotidien new yorkais répliquait "La France est une nullité dans le monde actif de la culture contemporaine" (G. ThOvERon, "Dallas, hélàs ! Mais Dinasty, nenni", La Revue Nouvelle, 1983, pp. 17-25).

3 Nous verrons ultérieurement comment les producteurs ne manqueront pas dans l'ultime épisode de la série de rendre un dernier clin d'œil à cet univers de référence sensible. 
sur J.R semble ${ }^{1}$ 'ailleurs avoir été annoncée en direct sur la plupart des vols internationaux, au même titre qu'un événement historique ${ }^{2}$. Fallait-il s'étonner dès lors que les déboires matrimoniaux de Sue Ellen se cautionnent de la figure de Jackie Kennedy et que la visite de Southfork Ranch semble avoir détrôné le pèlerinage à Elm Street dans les destinations touristiques?

Comme tout phénomène de société, le succès rencontré par Dallas intrigue et, rapidement, surgissent des questionnements aussi déroutants que paradoxaux. Comment par exemple, un produit si "typiquement américain" a-t-il pu connaître un succès international ? Mais aussi, comment un produit si "outrancièrement caricatural" -diront certains- a-t-il pu être à ce point apprécié aux USA ?

Il semble possible de ranger la majorité des travaux qui s'attachent à analyser le phénomène de son succès selon deux grandes perspectives : celle qui explique surtout le succès de la série par des stratégies de l'industrie de la culture et celle qui privilégie davantage la dimension symbolique de l'univers mis en place. Aux lectures proposées par ces perspectives et à leurs résultats, une troisième dimension nous semble devoir également être articulée pour rendre compte des diverses facettes du phénomène : la dimension d'une pragmatique ludique.

\section{La logique marchande}

Un des facteurs les plus fréquemment mis en avant pour expliquer le succès international de Dallas est la structure particulière du marché des industries culturelles dont il a émergé.

\section{La logique du dumping}

Pour certains, le succès de Dallas serait essentiellement le résultat éclatant des effets de l'inégalité des marchés culturels mondiaux et de la logique de véritable "dumping culturel" qu'il autorise : l'articulation d'une rentabilité domestique à une diffusion internationale.

1 G. SuFFERT, "Dallas : ah, les beaux salauds!", Le Point, ${ }^{\circ}$ 507, 7 juin 1982, pp. 120-121.

2 Faut-il encore rappeler que la suite de l'attentat contre J.R fut un des plus grands moments de l'histoire TV comme, en son temps, le fut la transmission mondiale de l'enterrement de John Kennedy? 
Ainsi, un épisode de Dallas, pourtant le plus cher de son époque, coûtait 1.960.000 \$1 à son producteur, la firme Lorimar. Le même épisode était vendu pour $1.960 .000 \$ 2$ à son diffuseur (CBS) et 300.000 \$ aux "syndicates" pour diffusion sur des réseaux locaux. La diffusion du même épisode engendrait pour CBS plus de 1.500.000 \$ de bénéfices publicitaires. Les gains domestiques, avant diffusion internationale, s'élevaient donc à $1.800 .000 \$$ par épisode.

Avec une telle rentabilité nationale, les producteurs peuvent se permettre de revendre la série en ajustant son prix international, non plus en fonction des coûts de production, mais des caractéristiques des marchés extérieurs ${ }^{3}$, afin de concurrencer plus efficacement les productions locales. Le prix de la série va donc s'étaler dans une fourchette allant de 1.000 à $62.000 \$^{4}$ tout en se démarquant jusqu'à $90 \%$ des barèmes (coûts de production et revenus publicitaires escomptés) d'une production locale ${ }^{5}$.

Certes une telle marge financière avec les productions locales aura facilité la diffusion internationale de la série, mais il faut cependant se garder d'en faire un facteur explicatif unique. De plus en plus les études proposent des bémols à un déterminisme de la logique marchande, soulignant d'autres éléments contextuels dans ce secteur. Ainsi, dans le cas de Dallas ne faudrait-il confondre ampleur de l'audience (aspects quantitatifs) et préférence culturelle (aspects qualitatifs). En effet, les plus hauts taux d'écoutes locales de la série se repèrent dans les régions de monopole télévisue ${ }^{6}$ et, dans les pays de concurrences télévisées, des produits locaux ont quelques fois détrônés Dallas? ${ }^{7}$ Dans le même ordre d'idées, les pays les plus

1 Dollars canadiens (données de 1986).

2 Ces données sont extraites de l'émission Télé-dollars.

3 Les revenus supplémentaires engendrés par ces droits de vente mondiaux s'élevaient en moyenne à $900.000 \$$ l'épisode.

4 Un épisode de Dallas a pu coûter 5.000 F.F. en Algérie, $1.000 \$$ au Pérou mais, au Canada $13.000 \$$ pour RC (francophone) et $62.000 \$$ pour CBC (anglophone).

5 Ainsi à Radio-Canada le prix d'un téléroman de qualité était estimé autour de 150.000 l'heure. Dallas s'offrait donc pour moins de $10 \%$ du prix local. La comparaison devient encore plus frappante si l'on se met à comparer les seuls investissements : acheter pour ce prix un produit de près de 2 millions, c'est, en quelque sorte, acheter " 10 fois plus pour 10 fois moins".

6 Par exemple Israël, Danemark, Algérie.

7 Ce fut le cas d'une émission de divertissement au Pérou et du téléroman Le temps d'une paix au Québec. 
réfractaires à la série Dallas se caractérisent aussi par une haute production locale ${ }^{1}$.

Si les caractéristiques de la logique économique rendent compte partiellement du succès des séries américaines en généra ${ }^{2}$, il reste encore à comprendre spécifiquement le succès atypique de Dallas.

\section{Un genre et son savoir-faire}

L'examen du contexte de production de la série nous conduit à croire que le succès de Dallas n'est peut-être pas l'effet d'un hasard mais l'aboutissement d'une recherche de repositionnement des industries culturelles américaines ${ }^{3}$. Pour nombre de spécialistes de la télévision américaine, Dallas fut bien à l'origine de la création d'un genre hybride : le "prime-time soap opera" ou le "grand soap opera"4. Dans une période (éphémère ?) de récession des auditoires américains, il s'agissait d'une tentative d'allier les acquis du soap avec les exigences du prime-time et donc, ce faisant, d'articuler les caractéristiques respectives du serial et des séries ${ }^{5}$. Ainsi, en conservant les règles nodales d'un univers interpersonnel et d'une structure narrative feuilletonesque, Dallas présentait nombre de démarcations par rapport

1 Par exemple, le Brésil et le Japon dont l'importation de programmes aurait chuté de 16 à $3 \%$ entre 1977 et 1986 selon Katz. Ajoutons également que si nous connaissons le nombre de pays où fut diffusé Dallas, nous ignorons ceux qui l'ont achetée au complet.

2 Ce qui nest par ailleurs pas nouveau, en 1972, plus de trente pays ont pu acheter pour moins de $100 \$$ chacun, les épisodes de la série Bonanza qui en coûtaient 250.000 à produire. (J. BIANCHI, "Dallas, les feuilletons et la télévision populaire", Études, févr. 1984, n³60/2, pp. 219-229).

3 Rappelons, à la suite de Bianchi (op. cit.), que les réseaux américains recoivent chaque année environ 7500 idées de séries, dont 500 deviendront des projets, 70 donneront lieu à un "pilote", 20 d'entre eux aboutiront effectivement à des séries dont 10 seulement survivront à la première saison de diffusion.

4 Pour être plus précis : suite à un essai du même Lorimar avec Peyton Place (19641969) mais qui restait l'importation simple du soap trois fois la soirée sans modification de la formule. Elle fut d'ailleurs reconduite en soap d'après-midi de 1972 à 1974. En outre, selon Katz, il faut noter que l'Amérique Latine et la GB semblaient déjà connaître des formules assez proches.

5 Les analystes anglo-saxons, pour faire court, s'entendent généralement pour désigner par le terme de "serial" les fictions de type feuilletonesque dont la trame est relativement "continue" (c'est-à- dire qui se poursuit d'épisode en épisode) alors que les "séries" se caractériseraient par des épisodes à la trame "discontinue" (chaque épisode étant relativement autonome l'un de l'autre). Le soap est, habituellement, un feuilleton de type serial, à la thématique largement sentimentalo-familiale et diffusé généralement au rythme quotidien d'une demiheure. 
au soap traditionnel. Il glissait d'un rythme de diffusion quotidien (le day time serial) à un rythme hebdomadaire, d'une heure d'écoute de l'après-midi à la soirée (et les modifications des exigences publicitaires), d'un budget restreint à des moyens financiers plus importants, de l'exclusivité de la topique sentimentale à un mélange des univers sentimentaux et d'action, de la sphère exclusivement privée à l'intrication du social sur le privé, de la domination du champ de la parole (la tradition du "radio-like opera") à une attention visuelle accrue.

On le voit, un tel projet de repositionnement du genre ne va pas sans une redéfinition des aspects formels du produit et les moyens investis vont, bien évidemment, permettre une "attractivité" plus grande de l'émission. Pour s'en convaincre il n'est que de citer la richesse des décors et des artefacts sur lesquels la presse internationale aura longtemps glosé, l'introduction plus systématique de scènes d'action et d'extérieur afin d'alléger les incontournables champs/contre-champs des échanges interpersonnels propres au soap et l'insertion régulière de dépaysements exotiques ${ }^{1}$. Les réalisateurs eux-mêmes insisteront sur le soin mis à la réalisation : "Nous filmons en $35 \mathrm{~mm}, 7$ à 8 minutes par journée alors que le soap médiocre peut parfois être obligé de tourner à trois caméras vidéo en temps direct"2.

Sur le plan narratif, Dallas va acquérir rapidement tempo vif et accéléré auquel la narration molle des soaps classiques ne nous avait guère habitués : alternant drame et détente, il propose -aux dires de ses auteurs- en un épisode ce que le soap quotidien mettrait des mois à raconter, en un an ce qui leur prendrait cinq années. Pour ce faire, la conception de l'épisode type sera articulée autour d'une vingtaine de séquences ${ }^{3}$, d'une durée maximale de deux minutes chacune, intégrant soigneusement le rythme des pauses publicitaires ${ }^{4}$ et développant pas moins de dix à onze trames narratives concomitament! Une

1 Vienne, Paris, les Caraïbes, la Colombie, Hong-Kong, par exemple.

2 Cité par S. Blum, "Dallas ou l'univers irrésolu", Réseaux, n 12, 1985, pp. 31-44. Ceci peut se repérer, entre autres, à quelques indices dont des mouvements de caméras relativement soignés pour le genre (mais qui, il est vrai, s'estomperont graduellement durant les dernières années de diffusion alors que, curieusement, pas moins de sept des acteurs s'essayeront alternativement au rôle de... réalisateur). Signalons également l'engagement d'acteurs particuliers pour ce type de production (la dernière épouse d'Elvis Presley, une comédienne de Hitchcock).

320 à 30 selon Ang. Dans un registre similaire, la série Lance et Compte qui s'inscrit dans la tradition visuelle ouverte par Dallas jouera autour des sept cents plans par épisode.

4 Prévoyant ainsi des points de tension particuliers aux séquences précédant la première, la seconde et la dernière des quatre pauses publicitaires prévues. 
telle densité narrative ne peut être opérationelle que si elle est supportée par des procédés de lisibilité maximale. On a pu relever ${ }^{1}$ dans Dallas le recours systématique à des motifs synthétiques (la scène du déjeuner ou du dîner, la réunion autour la piscine) qui permettent essentiellement au spectateur de "refaire le point de la situation". Signalons également la présence de codes de reconnaissance rapidement établis (la plongée sur Southfork, la contre-plongée sur le building, des bureaux de la compagnie ou le plan frontal sur l'habitat de Cliff Barnes, etc. ${ }^{2}$.

Deux autres caractéristiques du contexte particulier de la production de la série méritent d'être soulignés ici : la capacité d'adaptation aux réactions et l'entretien systématique du rapport au téléspectateur. Car ce savoir-faire à l'œuvre dans Dallas, se repère non seulement dans l'application des principes les plus éprouvés dans l'écriture et la réalisation, mais également dans sa souplesse d'adaptation aux réactions du public. On oublie trop souvent que le succès de Dallas ne fut pas instantané aux États-Unis. Cette émission fut initialement conçue comme une série (et même une mini-série) qui serait centrée autour du personnage médiateur de Paméla BarnesEwing. Or rapidement la réécriture des épisodes subséquents aux quatre premiers pilotes s'est orientée de plus en plus systématiquement vers une conception feuilletonesque du récit (en perdant donc la relative autonomisation des épisodes et même des trames principales) dont le "contre-personnage" de J.R. devient le moteur central. Cette attention au spectateur va être, dans le cas de Dallas, méticuleusement pris en charge tant à l'interne qu'à l'externe, à la fois dans le récit et dans son contexte de diffusion. Au-delà de la stricte nécessité narrative, apparaît un jeu systématique de la tension et du suspens qui tient compte des poses publicitaires, des fins d'épisodes mais aussi des ruptures de saisons. Le cas le plus probant aura été la diffusion de l'épisode révélant l'identité de l'assassin de J.R. L'impact de cet épisode (qui lui a valu la plus haute cote d'écoute fictive connue) aura été mis en place par la construction systématique de plusieurs culpabilités plausibles et l'arrestation de trois d'entre elles. Le tournage de la scène de la révélation a soigneusement été tenu secret: les participants n'ayant que des scenarii incomplets et plusieurs versions

1 Fl. DuponT, Homère et Dallas, Paris, Hachette, coll. "Les essais du XX $\mathrm{X}^{\mathrm{e}}$ siècle", 1990.

2 Avec, dès qu'on quitte ce paramètre très circonscrit, des récitatifs inscrits dans l'image "A Denver", "Vienne", etc. 
contradictoires ayant été captées. L'abondante publicité de cette discrétion durant les trois mois d'interruption inter-saisonnière ne faisant que redoubler systématiquement l'attente des 78 millions de spectateurs qui se retrouvent... 100 millions pour en voir le dénouement.

\section{La perspective sémantique}

Dans une perspective complémentaire à celle du contexte de production et de diffusion, nombre de critiques et de travaux vont s'attacher davantage au récit lui-même, aux motifs thématiques de l'univers présenté et à l'imaginaire auquel ils renvoient pour essayer de rendre compte du succès de ce feuilleton.

Un des éléments clefs de l'univers de Dallas, le plus évident et le plus régulièrement cité, est ce recours à la tradition de la saga familiale. Comme tout soap Dallas se construit sur le principe de 1'“interpersonnalisation" de l'univers ${ }^{1}$, mais il en présente l'application la plus classique : la famille dans sa dimension clanique, renouant ainsi avec une situation archétypique universelle remontant au-delà des particularismes culturels et favorisant donc des réappropriations locales diverses. L'essentiel des trames narratives du feuilleton vont décliner les aléas d'un tel projet fondateur : la rivalité clanique (entre les Ewing et les Barnes), les luttes matrimoniales autour des femmes, la quête d'une reconnaissance paternelle, les conflits au sein de la fratrie, les luttes de successions, la dimension incestueuse, l'interruption du lignage, la quête des descendants illégitimes, l'isolement des célibataires, etc. Autant de motifs séculaires, qui se retrouvent bel et bien au cœur de la plupart des mythologies culturelles particulières et dont les créateurs autant que les analystes rendent bien compte en référant régulièrement Dallas à l'opposition des Capulet et des Montaigu, à la Genèse (Caïn et Abel, Isaac et Ishmaël, Jacob et Isaïe) ou encore, pour demeurer dans la sphère américaine, A l'est d'Eden et, surtout, Giant ${ }^{2}$.

1 Disons, pour simplifier ce qui demanderait des développements plus nuancés, la reconstruction du monde autour du primat des relations interpersonnelles, excluant largement ce qui est "au-delà" et "en deçà" de cette sphère : les rapports strictement sociaux ou les situations d'introspection par exemple.

2 Roman d'Edna Ferber où s'entremêlent péripéties de générations pétrolières et quêtes de la légitimité filiale. 
Mais cette thématique universelle de la saga familiale sera cependant soigneusement inscrite dans un contexte aux dimensions mythiques et culturelles très localisées comme le laissait déjà évoquer la proximité des univers de Dallas et Giant. La construction patronymique du titre ${ }^{1}$ a valeur emblématique : derrière Dallas c'est le Texas, le western et l'imaginaire de "la dernière frontière" qui semblent convoqués. Dallas s'inscrit dans le mythe d'origine qu'aime se donner la nation américaine : l'esprit fondateur de quelques pionniers entrepreneurs, la valeur familiale et le passage de la tradition à la modernité (du ranch au pétrole). Certains soulignant d'ailleurs combien le projet narratif peut s'apparenter à ce genre mythologique qu'est le western. Si les coups mortels ne s'échangent plus dans les saloons mais par téléphone au sommet de tours luxueuses, l'enjeu demeure le même : un univers extérieur de luttes impitoyables pour assurer la survie de la cellule familiale, là "où les monopoles internationaux et les règles bureaucratiques n'auraient pas encore étouffé cette valeur fondamentale américaine de l'initiative individuelle"!

L'inscription au sein de cette mythologie se cautionnant par ailleurs de certains paramètres directement issus de la réalité sociohistorique. A l'époque du second embargo sur le pétrole arabe ${ }^{2}$ et à l'heure de son "président-cow-boy"3, la métropole Texane se perçoit comme le lieu d'un redéploiement industriel dans une économie américaine en récession, le lieu du rêve d'un nouveau départ, au croisement des oppositions traditionnelles de l'est et de l'ouest, du rural et de l'urbain, de la tradition et du moderne ${ }^{4}$. Parmi les commentaires qui cautionnent régulièrement le vraisemblable du feuilleton, il est mentionné que près d'un quart des cent plus grands promoteurs américains sont texans et que bien des Américains ont reconnu dans J.R. le personnage célèbre de H.L. Hunt"s. Certes, les quelques

1 Ainsi les Allemands ont préféré retitrer Dynasty sous l'expression : Le clan de Denver (E. KATZ et T. LIEBES, "Neither here nor there: japanese readings of Dallas", Communications, juin 1991, vol. 12, n², pp. 99-110).

1978.

3 Reagan élu en 1981.

4 La classique tension entre la capitale texane et sa "banlieue" rivale Forth Worth sera systématiquement balisée dans la série par la bi-polarité fondatrice des portions terrienne (Southfork) et pétrolière (les plans sur les gratte-ciel).

5 "Quand je vois Hunt bluffer le Congrès à la télévision, je le comprends mieux grâce à J.R.". Cité par Newcomb (op. cit.). Selon Libération (G. LEFORT, F. RousSEAU, "Dallas, ça recommence et c'est déjà fini", Libération, 18-19 sept. 1982, pp. 1822), Hunt, "joueur de poker professionnel, bluffeur de génie qui achetait ses 
mentions qui précèdent, demeurent extrêmement rapides et anecdotiques, elles indiquent cependant déjà comment spectateurs et critiques ont pu opérer des liens entre champ de la fiction et univers contextuel.

La réactualisation dans un contexte national d'une mythologie universelle ne suffit cependant pas à rendre compte de l'originalité de l'univers mis en avant par Dallas. Il n'est peut-être pas sans importance que ce feuilleton tourne essentiellement autour de cette figure très particulière de J.R. à la moralité familiale et professionnelle exécrable, figure maléfique de "celui qu'on aime haïr". Mais, ce faisant, Dallas n'est pas seulement un univers de transgression de l'ordre social. Certains travaux ont montré également comment il présentait une sensibilité sociale particulière à l'époque: la désillusion face aux grands garants institutionnels. Si l'on a pu comparer ${ }^{1}$ Dallas à une "version soap" du film Le parrain ${ }^{2}$ c'est en soulignant dans les deux cas, les hiérarchies familiales comme pivot central du récit, la présence des personnages omnipotents et cette persistance d'une reconnaissance paternelle et de sa vision du monde. Les deux œuvres construisent une dynamique très particulière de deux organisations aux pouvoirs illimités mais préoccupées par les liens du nœud familial. Dans les deux cas également on se trouve face à un imaginaire qui semble vouloir entériner dans les sphères de la culture populaire la fin du classique "le crime ne paie pas"3. Ce n'est donc pas à l'imaginaire du banditisme classique ou romantique de Bonny and Clyde 4 que Dallas se nourrit mais bien d'un contexte socio-culturel très daté qui va de l'affaire du Watergates, de la série des films à succès du Parrain, qui se poursuivra aussi avec Les Ripoux $^{6}$ et nous rejoint encore plus explicitement ces dernières

terrains pétrolifères à crédit" fut classé en 1952 comme le plus riche au monde. Mort en 1974, sept de ses descendants légitimes et illégitimes (nous restons bien dans l'univers de Dallas) sont cités par la revue Forbes parmi les plus riches des USA. A ne craindre les comparaisons par trop inégales, on pourrait avancer que Dallas fut à Hunt ce que Citizen Kane fut à Hearst !

1 M. S. MANDER, "Dallas: The Mythology of Crime and the Occult", Journal of Popular Culture, vol. 17, $\mathrm{n}^{\circ} .2,1983$, pp. 44-50.

2 The godfather de F. Coppola, sorti en 1972.

3 Ou, plus précisément, si "l'argent ne fait toujours pas le bonheur", il n'est plus vrai que "le crime ne paie pas".

41967.

51973.

6 Sorti en 1984. "Éloge du parasitisme, fresque cocasse du cynisme ambiant, Les Ripoux est un film politique. On y constate la faillite du pouvoir, le naufrage des 
années, ne fut-ce que par l'opération "Mains Propres" en Italie, le "scandale Agusta" en Belgique, les "Affaires" et la déchéance corrompue de Bernard Tapie en France. Si l'imaginaire du roman noir était traversé par un ressentiment contre la "société respectable", Dallas et Le Parrain traduisent davantage une désillusion sociale face aux organisations de la société libérale.

L'univers de Dallas, ce que Katz nomme sa dimension sémantique, semble ainsi reposer sur une articulation originale de trois niveaux: le recours à une strate archétypale de l'imaginaire, sa réappropriation au sein d'un cadre culturel déterminé et l'actualisation d'une sensibilité socio-historique ponctuelle' ${ }^{1}$.

\section{Vers une perspective pragmatique particulière}

Au-delà de ces deux perspectives et de la pertinence de leurs lectures, certains indices devraient nous stimuler à envisager également d'autres dimensions dans le succès de Dallas.

A relire différentes études sur la réception de Dallas $^{2}$, une tendance singulière peut se déceler en filigrane : plus les publics du feuilleton sont proches de la culture télévisée occidentale, plus la réception, même favorable, se fait de manière "distanciée". Cette perspective, nommée critique dans la terminologie de $\mathrm{Katz}^{3}$, se développe davantage au sein d'un public se réclamant de cultures "modernistes" plutôt que de cultures traditionnelles. Et dans les publics occidentaux, cette dimension est encore plus forte chez les publics des jeunes générations que chez ceux de la génération précédente $^{4}$. Ainsi c'est au sein du public américain que ce feuilleton sera pris le moins “au sérieux", qu'il sera appréhendé de la façon la moins

institutions. (...) Les Ripoux est une fable qui vaut bien des documentaires de télévision dits "d'information" écrivait Jean Collet (Études, mai 1985).

1 Au-delà du succès très particulier de Dallas, la confrontation de cette dernière hypothèse à d'autres succès médiatiques permettrait peut-être d'en jauger la pertinence heuristique.

2 Études très variables dans leurs ampleurs, leurs objectifs et leurs méthodologies, il est vrai.

3 Rappelons que dans sa recherche de base, Katz répartit les interventions des personnes interrogées selon leurs dimensions "référentielle" ou "critique". Il serait plus juste de dire que cette seconde dimension prend une place plus grande sans jamais dépasser la portion des interventions de type référentiel.

4 Cf., par exemple, M. H. Herzog , "Decoding Dallas", Society, n² 24, nov. 1986, pp. 74-77. 
référentielle. Certains mentionnant très clairement qu'à prendre Dallas au sérieux, on croirait que l'Amérique est essentiellement peuplée de pervers, alcooliques et corrompus ou encore que si 1'URSS avait produit ce feuilleton, elle aurait été accusée de la pire propagande simpliste.

Or cette démarcation -partielle, il est vrai-de ce type de public vis-à-vis de Dallas ne diminue pas nécessairement l'appréciation qu'il peut en avoir: on peut aimer Dallas malgré qu'on le trouve "invraisemblable"1. L'existence de cette attitude qui articule distance critique et appréciation doit nous conduire à aller voir au-delà des schématisations souvent trop simplistes de l'identification et de la mimésis projective pour comprendre le rapport qu'établit le spectateur avec Dallas. L'investissement spectatoriel pourrait prendre d'autres formes : on ne désire pas nécessairement devenir J.R. ou Sue Ellen !

\section{Du feuilleton au soap}

Interroger cet investissement spectatoriel, c'est revenir à un autre trait fondamental de Dallas, son caractère feuilletonesque et les propriétés particulières de ce type de récit. Dans le cas précis du téléroman, la tension entre les principes de résorption (tout s'arrangera bien un jour, les "bons" sentiments auront le contrôle des événements) et de procrastination (la résolution toujours reportée ${ }^{2}$ ) suscite et exacerbe un intérêt et un investissement que Katz appellerait de niveau syntaxique : l'attente, l'expectation, la supputation, le désir de savoir la suite maintenue systématiquement en suspens...

Mais le feuilleton télévisé possède deux caractéristiques qui permettent de le spécifier dans la pratique narrative du feuilleton en général et l'exploitation de ces deux dimensions peut, nous semble-til, faire basculer l'investissement syntaxique au profit d'un investissement davantage pragmatique. D'une part, il peut accéder à une certaine longévité (plusieurs centaines d'épisodes), offrir une densité d'écriture (jusqu'à une dizaine de trames simultanées), et s'inscrire dans une concurrence médiatique forte (qui oblige les produits à se

1 Ce qui est donc très différent des attitudes de rejet dans certaines contrées comme le Japon (E. KATZ, T. LIEBES, "Neither...", op. cit.). On peut même dire que le public le plus proche de l'univers de référence de Dallas est celui qui l'apprécie mais de façon plus distanciée.

2 Que ce soit dans le cas d'un feuilleton de type classique, "monotramé" ou sous les formes plus contemporaines du feuilleton télévisés "polytramé" : Dallas convoie pas moins de 10 trames narratives conjointement. 
démarquer, à faire "leurs marques"). Ainsi il se peut que l'originalité des situations et de leurs résolutions cède le pas à la récurrence, que la linéarité des grands classiques du feuilleton littéraire se colore de plus en plus de redites et de circularité. L'expectation du public n'y tient plus uniquement à la seule reconstruction de la plausibilité événementielle des épisodes précédents, elle relève davantage de la prévisibilité des motifs antérieurs chez l'auteur. Le plaisir n'y serait plus tant d'apprendre la suite que de se voir confirmer (ou infirmer) la suite ${ }^{1}$.

D'autre part, il existe une rupture fondamentale entre le feuilleton écrit et le serial télévisé, qui relève de la nature même du matériau de l'expression et qui vient renforcer cette idée d'un déplacement des attentes. Contrairement à l'univers livresque, le feuilleton télévisé se réalise à partir d'acteur en chair et en os dont les aléas personnels (la maladie, la mort) ou professionnels (la reconduction ou non du contrat, les exigences particulières concernant le développement du rôle proposé, etc.) sont connus et rapportés par la presse. Ce qui, dans le cas du film n'a qu'un rôle secondaire sur l'œuvre, peut, dans le cas du serial, devenir significatif. En effet, sur sa longue duree de vie et avec le développement d'une culture para-médiatique, cet aspect "extra-diégétique" devient une réalité intermédiaire entre l'auteur et le spectateur, celui-ci utilisant dorénavant ces informations dans sa "lecture" de l'œuvre : comment l'auteur va-t-il intégrer la fin du contrat de Patrick Duffy ou comment parviendra-t-il à nous faire avaler le retour d'un personnage déclaré mort ?

La présence de ces deux traits vient quelque peu déplacer le rapport du spectateur à l'univers du téléroman et lui donner une dimension pragmatique ${ }^{2}$ plus large. Les recherches récentes ont montré combien toute production média est tramée par un "contrat de lecture" implicite. Ce contrat comporte des dimensions propres au

1 "La série fait plaisir à l'usager parce qu'elle récompense ses capacités prévisionnelles : ce dernier est heureux de se découvrir capable de deviner ce qui se passera (...) ce n'est pas, néanmoins, nécessairement à la structure du récit qu'il m'attribue cette retrouvaille, mais bien à ses excellentes capacités prévisionnelles". U. Eco, "Le temps de l'art", in M. BAudson (dir.), L'art et le temps, Paris, Albin Michel, 1985.

2 Sans s'attarder outre mesure sur ce qui demanderait des développements plus raffinés, précisons que Katz reprend la classique trilogie des dimensions syntaxique, sémantique et pragmatique, cette dernière s'attachant surtout au rapport entre l'auteur et le spectateur dans un contexte de production particulier, et ferait partie de ce que certains nomment aussi le "contrat de lecture" de l'œuvre. 
genre auquel elle appartient ${ }^{1}$, au créneau de diffusion ${ }^{2}$, mais aussi à sa particularité même: la façon dont elle se démarque des autres produits concurrents (phénomène de singularisation). Dans le cas de Dallas, nombre d'indices laissent croire à une volonté délibérée de jouer sur ces contingences de la redite et de l'extra-diégétique pour établir un rapport particulier au spectateur, proche du registre ludique.

\section{Un espace clos}

Malgré le nombre impressionnant de ses épisodes, la galerie imposante de ses personnages et la relative complexité des intrigues, l'univers diégétique proposé par Dallas est un monde clos aux règles précises.

D'emblée ce qui frappe le spectateur de Dallas, c'est la façon dont il se singularise en transgressant deux des principes généralement admis du soap familial : les variations sentimentales doivent rester dans des limites décentes et le "mauvais" être extérieur au milieu familial central. Or ici, le pivot central n'est autre que J.R., personnage luciférien qui introduit une "nouvelle dimension narrative, l'exaspération"3. La formule qui est rapidement devenue sa publicité (Celui qu'on adore haïr) montre déjà le décalage par rapport une identification projective simple et l'introduction du recul.

Dallas s'inscrit aussi dans les schémas fondamentaux du récit mythique classique : la rupture de l'ordre initial (à l'origine le patriarche Ewing a dérobé femme et pétrole à son associé Barnes) et les luttes séculaires entre les deux clans jusqu'au retournement final (au dernier épisode, un Barnes possède le pétrole, et J.R. est dépossédé de sa femme) ${ }^{4}$. On a pu même montrer ${ }^{5}$ que les péripéties de cette saga s'organisaient autour de deux principes complémentaires : la recherche d'une fidélité (verticale) entre les générations et leurs descendances d'une part, la rivalité sans pitié au sein des

1 Dans le cas du téléroman on peut parler entre autres des principes de résorption (cf. supra) et d'interpersonnalisation (la réduction de l'univers aux logiques des relations interpersonnelles).

2 Pas de sexe, une violence modérée et la religion non explicite pour le prime-time de CBC.

3 J. BIANCHI, "Dallas...", op. cit.

4 Cf. annexe 1, schéma 1.

5 E. KATZ et T. LIEBES, "Dallas and Genesis : Primordiality and Seriality in Television Fiction", in J. CAREY (ed.), Media, Myths and Narratives, Sage, 1987, pp. 113-125. 
fratries. Une lecture attentive de Dallas permet cependant de repérer une logique beaucoup plus précise encore dont les règles ne pouvaient manquer de devenir de plus en plus apparentes aux spectateurs fidèles. Ainsi, l'ensemble des événements qui relancent régulièrement la saga, les éléments clefs à la base des intrigues et des trames narratives, ce qui constitue de fait le paradigme des "menaces" à la paisibilité de l'univers familial, se révèle très étroit et très déterminé. L'univers de Dallas s'alimente en effet largement à ce qui semble le parcours systématique et récurrent des transgressions du modèle d'ordre familial dominant. Un relevé des intrigues, même fragmentaire, permet notamment d'en repérer quelques traits.

Au-delà des nombreuses relations sentimentales "classiques" des deux principaux personnages ${ }^{1}$, on retrouve dans Dallas, en élargissant la définition de la relation incestueuse aux oncles, aux beaux-frères et belles-sœurs au moins dix occurrences sur ce thème. Bien davantage encore, si on élargissait la catégorie à la rivalité entre père et fils pour la même conquête amoureuse ${ }^{2}$.

Tout aussi caractéristique, sur les quinze "enfants potentiels" de la troisième génération dont nous parlera la saga, aucun ne vivra entre son père et sa mère légitimes et nous assisterons à la déclinaison des variations de la filiation problématique ${ }^{3}$. A ce titre le tableau des rapports de J.R avec sa descendance est un véritable cas d'espèce : (Annexe 1, schéma 3).

Signalons encore comment le principe d'élargissement de l'univers semble se faire par une accumulation systématique de situations de réapparition des "familles dans le placard". Un nombre impressionnant de ces nouveaux personnages qui viennent régulièrement se greffer au récit au cours des épisodes proviennent de la sphère familiale élargie, et toujours dans une relation secrète et souvent perturbée. Ainsi plus de trente-trois nouveaux membres familiaux vont venir s'ajouter (frères, sœur, père, demi-frère, ancienne épouse, ancienne relation...), soit pas moins d'une occurrence tous les quinze épisodes. Étudiant le feuilleton littéraire et la littérature

1 Qui au passage, en terme de récurrences, se permettent d'épouser-divorcer et réépouser et reperdre leur première femme.

2 Cf. annexe 1, schéma 2.

3 Une fausse couche, une grossesse feinte, une mort-née, dix paternités non reconnues, dont certaines "incestueuses" (avec des belles-sœurs), un abandon, une adoption qui échoue, etc. La seule -heureuse- exception est une reconnaissance de paternité... au tout dernier épisode. 
populaire du $\mathrm{XIX}^{\mathrm{e}}$ siècle, Umberto Eco a déjà souligné l'importance cardinale du motif de l'agnition ${ }^{1}$. Cet artifice narratif, réduit à la sphère du familial, est un des moteurs réguliers de la série. Le cas de Jamie (schéma 4) illustre bien ce principe de "cooptation familiale" : son intégration au récit (en 1984) va successivement entraîner la "création" de son père, un frère ignoré de Jock, de son propre frère (1985), de la femme de celui-ci (1986), de la sour de cette dernière (1987), etc., chacun de ces nouveaux personnages impliquant de facto une redistribution complète des rapports financiers et sentimentaux du feuilleton.

Cette description de la singularité de l'imaginaire de Dallas $^{2}$ ne vise pas à suggérer un attrait particulier d'un tel univers (qui justifierait un investissement sémantique) mais vient plutôt souligner la prévisibilité qu'elle induit (qui elle, releverait davantage d'un investissement pragmatique).

\section{Le jeu avec les conventions}

Parallèlement à cet imaginaire relativement rigide, Dallas va se permettre une série d'écarts aux standards du vraisemblable téléromanesque afin d'intégrer diverses réalités et pressions extradiégétiques.

On peut relever, par exemple, nombre de marques qui vont à l'encontre de l'homogénéité de l'univers présenté et demandent donc au spectateur un élargissement du contrat de vraisemblable : on assiste à un changement important de décor pour Southfork entre les quatre premiers épisodes et la suite de la saga, trois personnages vont changer d'acteur durant les saisons, un personnage se payant même

1 "L'agnition : note pour une typologie de la reconnaissance", in De superman au surhomme, Paris, Grasset, 1993, pp. 29-38. L'auteur y présente l'agnition comme la reconnaissance par un personnage -ou par le lecteur- de l'identité réelle d'un autre personnage. Le topos du "faux inconnu" en est une des versions les plus fréquentes. Dans le cas de Dallas, il s'agirait surtout d'agnition contrefaite.

2 Notons que le roman, écrit légèrement après le début de la série, vient cautionner et accentuer ces dimensions en tranchant là où subsistait un doute (John Ross n'est pas le fils de J.R.), en explicitant des implicites (Lucy est la fille de J.R) et en inventant, rétrospectivement, une justification générique du rapport de J.R aux femmes (une relation malheureuse qu'adolescent il aurait eue avec une femme adulte : là aussi on retrouve un retournement de situation dans sa relation avec la jeune Cally). Par ailleurs, il n'est peut-être pas inopportun de rapprocher l'existence d'études structurales (au sens "Levi-Straussien" du terme) de Dallas à cette clôture très stricte de son imaginaire (cf. notamment les travaux de Baier). 
trois actrices successives ${ }^{1}$; enfin un des personnages principaux (Miss Ellie) est joué temporairement par une tout autre actrice, le temps d'une saison (et de la maladie de l'autre) ${ }^{2}$.

Mais suivre Dallas aura aussi exigé de la part de son spectateur un élargissement de la "plausibilité diégétique". Autour des contingences internes (la relance du récit) et externes (l'interruption de contrat ou la mort des acteurs par exemple), cette série nous aura donné une déclinaison systématique des stratégies narratives face à la disparition et à la réapparition des personnages ${ }^{3}$ au point d'y jouer avec le spectateur par presse interposée. Poursuivant les réflexions d'Eco sur le motif de l'agnition, il est tentant -et plaisant-, dans le cas de Dallas, d'avancer le terme de "réagnition" pour désigner ces artifices narratifs qui visent à réintroduire -et donc à faire reconnaître- un personnage diégétiquement disparu. A ce sujet, Dallas peut se présenter comme une véritable anthologie des procédés narratifs qui permettent à un auteur de réintroduire un personnage diégétiquement disparu. N'en citons que quelques uns. Dallas sera sans doute la seule série à véritablement ressusciter quatre personnages diégétiquement morts ${ }^{4}$. L'explication est alors celle de la "fausse mort" dans un accident qu'on s'était bien gardé de révéler plus tôt afin de ne pas faire souffrir les proches qui ont refait leur vie, ou encore pour échapper à un jugement. Peut-être en réaction à ces réintroductions "brutales" on pourrait citer les "manœuvres préventives" qui permettent d'utiliser ultérieurement l'événement de diverses manières: Pam disparaît du feuilleton, sans que jamais Bobby ou nous ne sachions pourquoi, ni où (on pourra donc réutiliser éventuellement le personnage); de même nous n'avons jamais vu l'image du cadavre de J.R après son suicide final (une suite à Dallas n'est donc pas à exclure). Mais le personnage disparu peut aussi revenir sous forme de sosie et il faudra à Bobby (et au spectateur) près d'une saison pour s'assurer que ce n'était pas Pam qui le hantait mais une personne très semblable physiquement. Le plus simple reste évidemment de proposer un successeur au personnage disparu

1 Jenna Wade jouée successivement par Morgan Fairchild (78), Francine Tacker (80) et Priscilla Presley (83-88). Ces changements d'acteur ne sont bien sûr en rien justifiés par un "vieillissement" du personnage.

2 Avec cependant la même voix, pour ceux qui suivent les traductions...

3 Transgressant par là des règles de plausibilité internes implicites au genre telles que celles que Van Dine avait pu énoncer pour le roman policier : pas de jumeaux, etc.)

4 Il s'agit de Dusty Farlow, Kristin Shepard, Mark Graison et Bobby Ewing. 
suffisamment proche pour endosser tel quel le rôle et en reprendre les fonctions narratives propres: Jock meurt, on trouve un nouvel amoureux assez proche dans sa manière : Clayton Farlow. Une autre variante du genre est celle qui prévoit l'apparition du remplaçant physique : le personnage revient mais physiquement transformé (le nouveau contremaître à Southfork ne serait-il que Jock Ewing méconnaissable suite à des chirurgies dues à ses brûlures ?). Même mort, un personnage peut rester narrativement actif : il s'agit alors de développer une stratégie du "fantôme" : Jock est mort mais on lui invente un passé insoupçonné : il agit encore à distance par vieilles lettres interposées ${ }^{1}$. On a déjà évoqué le procédé de substitution : un autre acteur reprend momentanément le rôle (Miss Ellie) le temps d'une maladie par exemple. Mais ce tableau resterait incomplet si on ne rappelait l'artifice le plus "audacieux" qui aura marqué Dallas. Désireux de réintroduire le personnage de Bobby bien qu'étant déclaré mort dans un accident automobile, la production a joué avec un appel public de "solutions" pour simplement oser proposer... un simple cauchemar de son épouse qui couvrirait une période diégétique assumée par pas moins de 31 épisodes !

Ici non plus il ne s'agit d'essayer d'expliquer l'attrait du feuilleton par la présence de ces procédés, l'accumulation de telles coïncidences étant rarement le critère d'une qualité particulière. Par contre ces élargissements de la plausibilité permettent de mieux comprendre le glissement qui s'opère peu à peu dans la relation au spectateur, la dimension très particulière du rapport pragmatique proposé par Dallas. Par exemple, comme tout épisode de feuilleton classique, l'épisode 223 s'achève sur un suspens. La dernière séquence de l'épisode montre J.R. murmurer qu'il faut s'occuper rapidement d'un personnage qui semble vouloir le contrarier. Ce suspens, déposé explicitement par les auteurs, crée chez le spectateur cette expectation propre au récit feuilletonesque, une attente que Katz nommerait syntaxique. Or pour un spectateur assidu, la véritable attente n'est pas créé par cette dernière séquence (on a compris que J.R. va nous mijoter un mauvais coup dont il a l'habitude) mais dans l'avant-dernière, là où Ray, contremaître au ranch des Ewing, annonce qu'il va se lancer dans sa propre entreprise en précisant qu'il trouvera un remplaçant fiable. Et la matriarche Ewing de bien insister : "Oui, oui je sais que tu nous trouveras quelque de très bien". Dialogue explicitement

1 La quête de Pam en Colombie tient également à une manœuvre de ce type. 
anodin mais qui conduit un spectateur de Dallas à supputer que le projet de Ray est sans doute un artifice des auteurs afin de ménager la place pour un nouveau personnage dans le feuilleton. En soulignant les qualités nécessaires du futur candidat et en connaissant la propension du feuilleton pour ces "familles dans le placard" et les phénomènes de "réagnition" le spectateur se met à jouer avec l'identité du nouvel arrivant ${ }^{1}$. Ce faisant l'attente n'est plus de la même nature, elle n'est pas supportée par la seule dimension diégétique mais par une connaissance des règles implicites de la construction de cet univers : le classique "suspens syntaxique", se double d'un "suspens pragmatique". L'accentuation du rapport pragmatique acquiert donc, dans Dallas, une dimension notablement ludique.

Une telle hypothèse se confortera encore des nombreuses situations qui se donnent à lire comme autant de clins d'œil aux conventions narratives. Pour faire court, qu'il nous suffise d'en énumérer quelques types.

L'incipit de l'épisode d'ouverture n'avait-il déjà pas l'allure d'une citation détournée, rappelant, en la retournant, les happy ends des films hollywoodiens des sixties. Là où, au terme de péripéties diverses, les jeunes mariés quittaient leurs familles attendries et s'éloignaient confiants sur l'autoroute de leur bonheur, le feuilleton réplique en débutant par l'arrivée sur l'autoroute de ce jeune couple de mariés, inquiet de la réaction mitigée de leurs familles respectives et des péripéties qui peuvent s'en suivre ${ }^{2}$.

A diverses reprises le feuilleton s'est permis des mises en abîme de plus en plus précises sur l'univers de la télévision et même du téléroman. Le premier épisode toujours, nous introduisait aux affaires de la compagnie Ewing par télévision interposée : on assistait, avec J.R. ${ }^{3}$ à la retransmission en direct de la commission sénatoriale. Ces allusions à la réalité de l'univers de la télévision, reviendront épisodiquement tout au long du feuilleton: plus tard, la seule ruse que trouvera Sue Ellen pour empêcher définitivement J.R de chercher à lui nuire sera de lui annoncer qu'elle a écrit, produit et mis sous scellés

1 Qui, de fait, se révélera être, le temps d'une saison, le possible retour du patriarche déclaré mort mais peut-être défiguré.

2 C'est bien pourquoi le rapprochement thématique -toutes proportions gardées- de Dallas et de Roméo et Juliette ne se comprend que si on considère l'un comme l'alternative de l'autre : et s'ils s'étaient épousés que se serait-il passé ?

${ }^{3}$ Lors de cette première apparition, le personnage était évidemment de dos redoublant ainsi dans l'écran la position du spectateur que nous étions à ce moment : tous deux nous regardions Dallas à la télévision. 
une fiction télévisée présentant ses odieuses manigances : rien d'autre donc qu'un Dallas dans Dallas. Le dernier épisode de la série ne fera pas moins que de présenter une longue séquence mettant en scène la production d'un téléroman avec ses arrière-fonds de négociations financières et de rivalités entre actrices cautionnant ainsi les données extradiégétiques que rapportaient régulièrement la presse spécialisée à propos de Dallas lui-même.

Mais Dallas a pu jouer aussi de l'effet de détournement des conventions narratives. Par exemple le personnage d'April Stevens meurt au cours d'un épisode de la dernière saison. Mais le générique du feuilleton qui est constitué de très brefs extraits d'épisodes divers, continue à montrer une apparition d'April avec un nouveau-né alors qu'elle n'a pas encore eu d'enfant. Le spectateur qui sait donc n'avoir pu voir cette scène dans le déroulement de l'histoire, et qui s'est habitué aux phénomènes de réapparitions propres à Dallas, attend et guette les signes précurseurs de sa réintroduction. De fait, dans la saison qui suit, nous aurons droit successivement à une vision muette (une illusion due à un sosie), puis une illusion parlante (mais c'était un rêve) et enfin la séquence en question qui, par un fondu enchaîné, se révélera être une image projective faite sur une autre femme. En insérant ainsi au sein du générique du fictif fantasmé ${ }^{1}$, l'auteur ne propose rien d'autre qu'un détournement du rapport pragmatique instauré par la série, un jeu sur le jeu.

Cette mise en avant des conventions narratives, n'aura peut-être jamais été aussi directement explicite que dans l'ultime épisode de la série et sa perspective nettement auto-parodique. Cet épisode était essentiellement a-typique à la série et en rupture avec tous ceux qui l'ont précédé. D'une durée exceptionnellement longue, il introduisait des éléments nettement fantastiques dans un univers qui se voulait jusque-là exclusivement réaliste ${ }^{2}$. En outre il était, en quelque sorte, diégétiquement inutile : entre la dernière image de l'épisode précédent et l'ultime image de la série, rien de "nouveau" ne se sera passé. Il serait même malaisé de soutenir que l'épisode puisse motiver le coup de feu du suicide. Par contre, dans cet épisode de deux heures les auteurs semblent s'amuser à déconstruire systématiquement nombre de conventions antérieures propres à la série elle-même. Recourant à

1 Du "fictif-fictif" parmi du "fictif-réel" en quelque sorte.

2 D'une durée de deux heures au lieu d'une, il mettait par exemple en scène un personnage d'ange-démon permettant de se rendre invisible et d'entrevoir une réalité alternative. 
l'artifice narratif de cet être étrange qui permet à J.R de visualiser ce qu'aurait été l'univers de Dallas sans sa présence maléfique', le feuilleton va ainsi nous proposer une suite de séquences où les personnages de Dallas nous sont présentés dans des trajectoires voire des personnalités radicalement différentes de celles auxquelles quatorze saisons d'écoute nous avaient habitués. Il y a un effet extrêmement déroutant de voir ainsi Gary le faible devenir subitement un homme aux succès professionnels et sentimentaux éclatants; le bon, le loyal et vertueux Bobby se transformer en joueur compulsif lâche, veule et irresponsable. Ces diverses séquences intégrant d'ailleurs des effets de surprises ou de clins d'œil ${ }^{2}$. Ce qui est mis ainsi à nu c'est, bien sûr, la confusion entre acteur et personnage qui supporte toute fiction mais aussi l'infini et l'arbitraire des possibles narratifs dans un tel univers. Nous assistons avec les auteurs à l'évocation de tous ces "téléromans" auxquels nous avons échappé. Cette activité autoparodique prendra une tournure véritablement jubilatoire dans cette séquence déjà évoquée où Sue Ellen joue le rôle d'une actrice. Non seulement on y réintroduit l'univers de la télévision dans la télévision et même des conditions de production de la fiction téléromanesque mais cette séquence présente une habile caricature de l'écriture romanesque et de ses procédés tels qu'ils furent mis en œuvre dans les centaines d'épisodes de Dallas. Dans une série étourdissante de retournements de situations, cette séquence joue littéralement avec la perception du spectateur pour qui rien ne peut plus être tenu pour définitif : dans un premier temps, Sue Ellen y est présentatrice vedette au faîte de sa notoriété (perception valorisante de la situation du personnage $:+$ ) mais qui est contrainte de laisser la place à une plus jeune compétitrice (retournement : la situation apparemment heureuse est, en fait, malheureuse : -). Un recul de caméra nous montre que tout cela n'est qu'une situation d'un téléroman qui se tourne et que si Sue Ellen quitte la série, c'est pour un projet de film plus valorisant (retournement de situation : + ), sauf

1 Notons qu'il s'agit donc d'une situation d'uchronie par rapport à un univers premier qui est déjà fictif par lui-même.

2 Ainsi dès que la logique du retournement simple (le bien en mal, le mauvais en bon) devient trop prévisible, d'autres variations apparaissent : la surenchère : Cally, femme malheureuse avec J.R, aurait pu être... encore beaucoup plus malheureuse avec un autre ; Cliff, l'éternel perdant semble gagner et même subitement devenir... président des États-Unis (relançant sur le mode parodique la proximité des deux univers) ou les compromis : Ray aurait été plus minable (professionnellement) et plus heureux (familialement), etc. 
que ce projet de film se révèle, dès les minutes qui suivent, un projet bidon qu'elle doit rejeter (retournement: -), alors que son mari lui annonce avoir abandonné son emploi pour pouvoir la suivre lors de son tournage (situation aggravée: --). Mais leur liberté professionnelle leur semble une occasion inespérée de vivre quelques moments de leur bonheur conjugal (retournement $:+)^{1}$.

\section{La pragmatique ludique du téléroman}

Au terme de ces quelques digressions, le succès exceptionnel de Dallas nous semble redevable de divers facteurs, de nature et d'effets complémentaires. La situation économique particulière du marché des biens culturels et le recours à un univers basé sur un imaginaire qu'on a parfois pu qualifier d'universel auront dû aider à la diffusion internationale de la série. L'inscription de cette thématique dans des codes nationaux, la présence d'une sensibilité très ponctuelle à l' "air du temps" dans un produit à la mouture technique et formelle rehaussée, rend probablement compte d'une partie de son succès dans les publics nord-américains et occidentaux. Mais l'appréciation de cette série par un public qui la perçoit pourtant largement comme caricaturale et sans grande vraisemblance nous conduit à souligner la singularité de son rapport au spectateur. A l'instar de toute production feuilletonesque, Dallas doit tenir compte de la relative prévisibilité de ses agencements et de ses effets sur la nature de l'attente du spectateur. Loin de nier cette dimension, la série semble au contraire avoir réussi à la prendre en charge sur le mode d'une relation proprement ludique qui nous fournit peut-être quelqu'indication pertinente sur les tendances à l'œuvre dans la culture médiatique contemporaine.

1 On notera également dans cette séquence la mise en abîme de l'action de l'extradiégétique sur l'univers diégétique: Sue Ellen désireuse de quitter le téléroman où elle joue, celui-ci doit prévoir une péripétie impliquant sa disparition de la diégése. 
Annexe 1

Schéma I : la rupture fondatrice de l'univers de Dallas

\section{Les paramètres originaux}

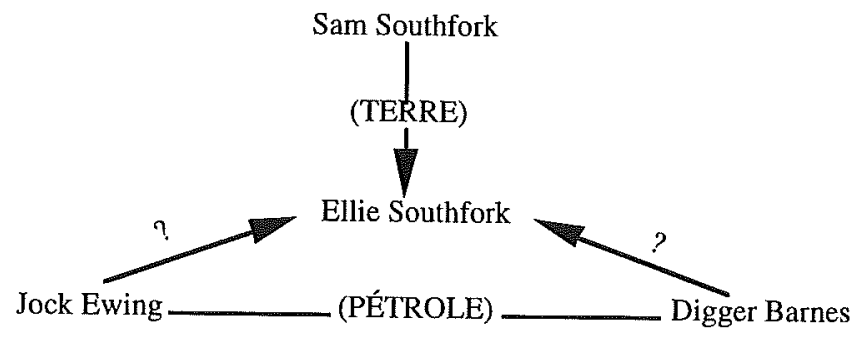

\section{Le déséquilibre initial}

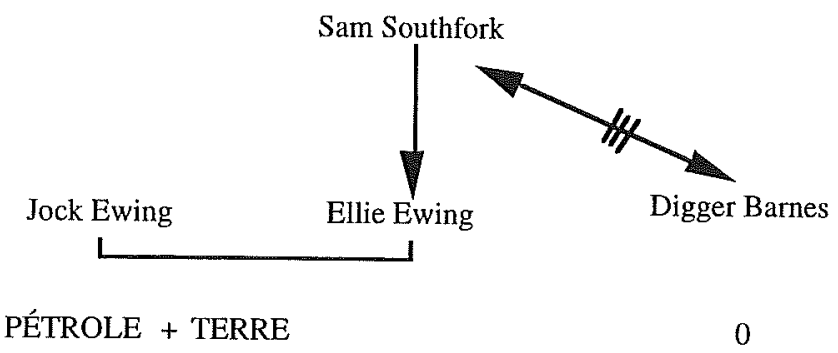

A l'origine de Dallas, un déséquilibre fondamental. Jock et Digger copropriétaires de champs pétrolifères soupirent après la main d'Ellie, héritière des grandes propriétés de son père. Digger séduit l'héritière mais trahit une promesse de celle-ci à son père en forant sur les terres ancestrales. Révolté, Jock parviendra par une manœuvre douteuse à évincer son rival dans le cœur d'Ellie et dans la propriété des pétroles. Le couple Jock et Ellie Ewing se trouve ainsi triplement valorisés du bonheur conjugal, de la possession de la terre et des profits pétroliers tandis que Digger Barnes se retrouve dépouillé de tout. Ce déséquilibre initial mettra 357 épisodes à se rétablir. 


\section{Schéma 2 : les généalogies}
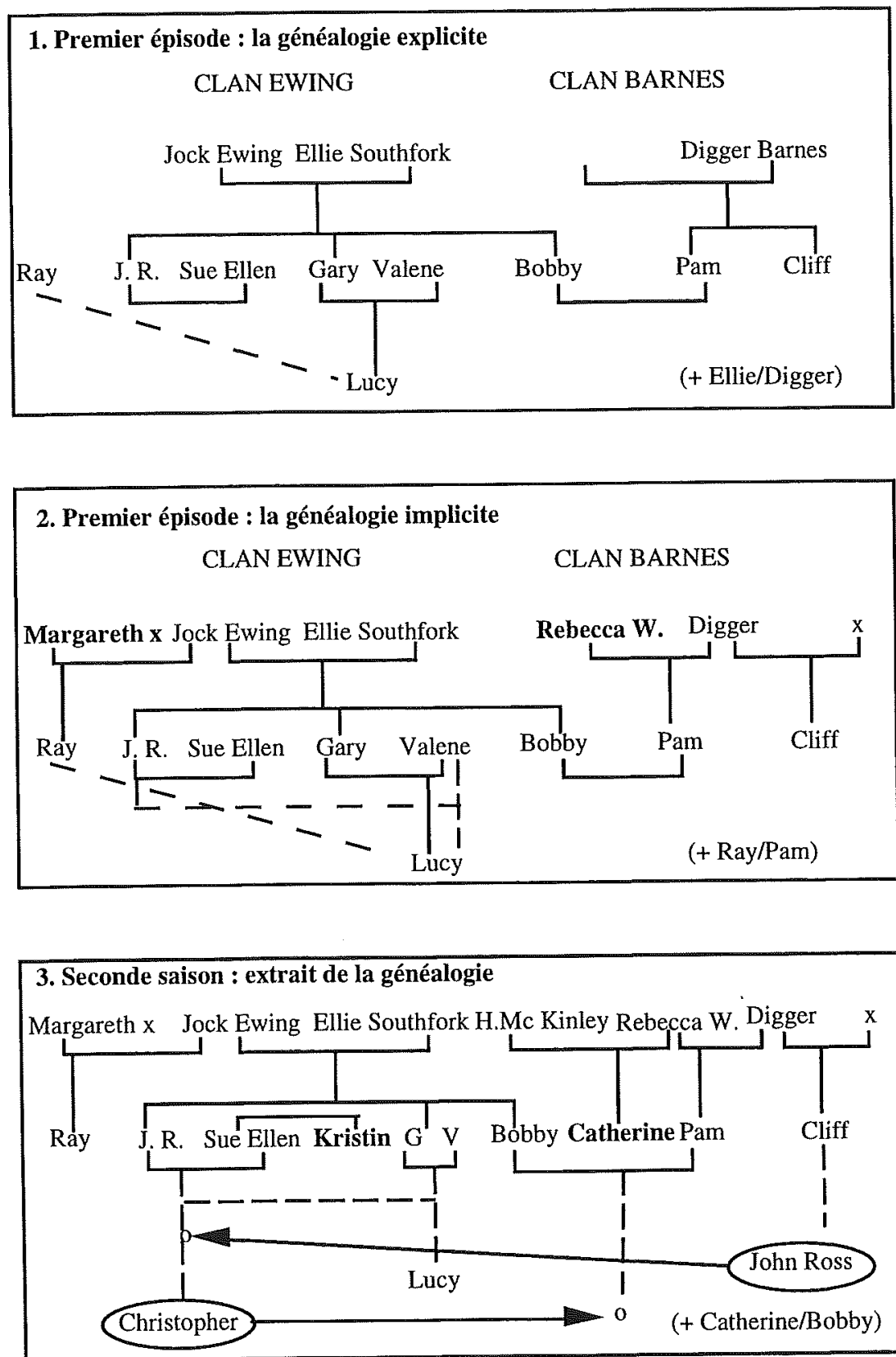
Ainsi, derrière la généalogie très classique du premier épisode, se cachaient déjà trois filiations implicites : paternités non reconnues pour Ray et Lucy, et maternité non reconnue pour Pam. Ce faisant, Lucy entretient une relation sentimentale avec son oncle. Une saison plus tard, le tableau se corse encore davantage en introduisant des sororités non reconnue (Catherine) ou ignorée (Kristin) et deux enfants détournés de leurs pères : John Ross, fils de Cliff est élevé par J. R., tandis que Christopher, fils de J. R. avec sa belle-sœur, sera élevé par Bobby et Pam.

\section{Schéma 3 : Dallas, la descendance problématique : (déclinaisons sur) le cas $J . R$.}

1. J. R. renie Lucy, l'enfant qu'il a eu avec sa première belle-sœur (Valene).

2. Il se croit géniteur d'un enfant (John Ross) de sa première épouse (Sue Ellen), mais qui est de son ennemi.

3. Il ignore l'enfant (Christopher) qu'il a eu avec sa seconde belle-sœur (Kristin) et qui sera adopté par son frère Bobby.

4. Il a cru avoir un descendant (grossesse feinte) de sa seconde épouse Cally.

5. Il se découvre un fils de 20 ans (James) d'une ancienne relation (Vanessa Beaumont). Ce fils flirtera avec sa belle-mère Cally et se découvrira luimême un fils ignoré (Jimmy).

6. Il voit lui échapper son seul enfant "légitime" (de Cally) lorsqu'elle le quitte, enceinte, pour un autre. 
Schéma 4 : la narration par "cooptation" familiale :
"la preuve par Jamie"

\section{Phase initiale}
Clan Ewing
Intrusion

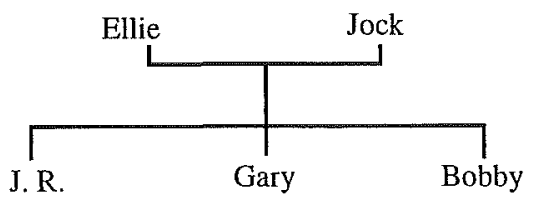

$?$

Qui est cette Jamie?

\section{Phase développée}

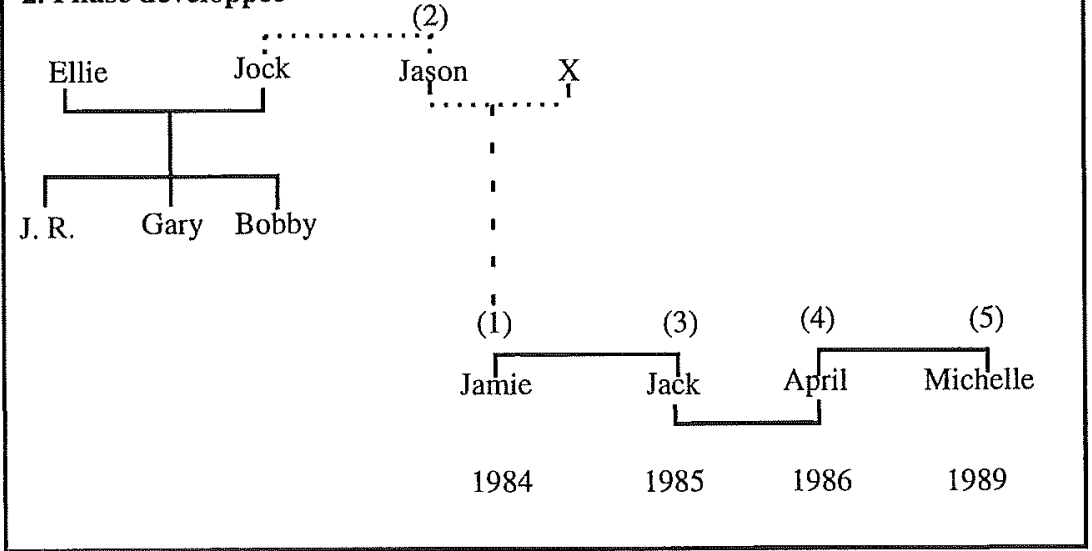

L'introduction du personnage de Jamie en 1984 aura donc entraîné la création d'un frère oublié de Jock, puis un frère Jack (1985), l'ex-épouse de celui-ci, et la sœur de cette dernière, ceci sans parler de leur mère, et d'un enfant problématique. Chaque apparition permettant une redistribution complète des prétentions à la propriété des actions de la compagnie Ewing et une nouvelle combinatoire des relations sentimentales. 
Annexe 2

\section{Éléments bibliographiques concernant Dallas}

\section{Monographies}

ANG, Ien, Watching Dallas. Soap opera and the melodramatic imagination, London and New-York, Methuen, 1985, $148 \mathrm{p}$.

BIANCHI, Jean, Comment comprendre le succès international des séries de fiction à la télévision? (Le cas "Dallas"), Paris, Ministère de l'industrie et de la recherche, Laboratoire CNRS/IRPEACS-Lyon Ecully, 1984, 101 p.

Dupont, Florence, Homère et Dallas, Paris, Hachette, coll. "Les essais du xx siècle", 1990, 169 p.

LAEBES, Tamer et KATZ, Elihu, The export of meaning. Cross-cultural readings of Dallas, Oxford University Press, 1990, 188 p.

\section{Articles, rapports et chapitres de livres}

ALASUUTARI, Pertti, "I'm ashamed to admit but I have watched Dallas : The moral hierarchy of television programmes", Media culture and society, vol. $14, n^{\circ} 4$, oct. 1992, pp. 561-582.

ANG, Ien, "Melodramatic identifications: television and women's fantasy", in BRown, Mary Ellen (ed.), Television and women's culture: the politics of the popular, London, Sage, 1990 , pp. 75-88.

ARLEN, Michael, "Smooth pebbles at Southfork", in ARLEN Michael (ed.), Camera age : essays on television, New-York, Farrar, Strauss and Giroux, 1980, pp. 38-50.

Augé, Marc, "Héros téléculturels ou une nuit à l'ambassade", Le temps de la réflexion, $\mathrm{n}^{\circ} 4$, Paris, Gallimard, 1983.

BAIER, Michel, "La pensée sauvage de «Dallas»", Le Nouvel Observateur, n 1037 , 21 sept. 1984, pp. 62-66.

BAIER, Michel, "La morale de Dallas", Les Temps Modernes, sept. 1984, pp. 590616.

BANG Jorgen, "Reception Research and Studies of Culture. On Quantitative and Qualitative Approaches in Danish Media Research", Nordicom-Review-ofNordic-Mass-Communication-Research, $\mathrm{n}^{\circ}$ 1, 1988, p. 14-20.

BIANCHI, Jean, "Dallas, les feuilletons et la télévision populaire", Études, n' 360/2, févr. 1984, pp. 219-229.

BiANChI, Jean, "La promesse du feuilleton", Réseaux, 1988, pp. 7-18.

BIANCHI, Jean et CHAGNIAC, Régine, "Feuilletons et séries à la télévision française", Généalogies, Bry-sur-Marne, INA-IRPEACS, 1989.

BLum, Sylvie, "Dallas ou l'univers irrésolu", Réseaux, n 12, 1985, pp. 31-44.

BONDEBJERG, Ib, "Den elektroniske fiktion : Aestetik, fascination og ideologi i TVserierne Dallas og Heimat (La fiction électronique : esthétique, fascination et idéologie dans les séries TV)", Kultur-og-Klasse, vol. 15, n4 (60), avril 1988 , p. $54-86$. 
BROWER Susan, "TV 'Trash' \& 'Treasure': Marketing Dallas \& Cagney and Lacey”, Wide-Angle :A Film Quarterly of Theory Criticism and Practice, vol. 11, $\mathrm{n}^{\circ} 1$, pp. 18-31.

CANTor,Muriel et PINGREe, Suzanne, Soap opera, Beverly Hill, Sage, 1983.

CollectiF, "Dossier Dallas", Les deux écrans, n 39, nov. 1981.

Collins, Ava, "Intellectuals, Power and Quality Television", Cultural-Studies, janv. 1993, vol. 7, n 1, pp. 28-45.

CoRnu, Gérard et ChaPelaIN, Brigitte, "Dallas : le feuilleton universel, in L'état du monde 1983, Paris, La Découverte-Maspéro, 1983, pp. 566-568.

EMANuEL, Susan, ANG, Ien, "Watching Dallas : Soap Opera and the Melodramatic Imagination", in BARKER, Martin, BEEZER, Anne (ed.), Reading into Cultural Studies., London, Routledge, pp. 21-33.

Herzog Massing, Herta, "Decoding Dallas", Society, n² 24, nov. 1986, pp. 74-77.

HIRSCHMAN Elizabeth C., "The ideology of consumption : A structural-syntactical analysis of «Dallas» and «Dynasty»", Journal of Consumer Research, déc. 1988 , vol. $15, n^{\circ} 3,1988$, pp. 344-359.

HIRSCHFEld, Burt et RAINTREe, Lee, Les Maîtres de Dallas, Les Femmes de Dallas, Les Hommes de Dallas, Paris, Éd. J'ai Lu, 1981.

HJoRT Anne, When Women Watch Television: How Danish Women Perceive the American. Serie "Dallas" and the Danish Serie "Daughters of the War", Copenhagen, Media research departement, Danish Broadcasting Corporation, 1986.

Hood, Williams John, "Soaps and the Sociology of the Family", Teaching-Sociology, vol. $14, n^{\circ} 4$, oct, pp. $270-272$.

JoHnson, S. "Dallas Ø/Dallas", Southwest Review, USA, vol. 70, n 1, pp. 31-42.

KATZ, Elihu et LIEBES, Tamar (1980), "Patterns of involvement in television fiction : a comparative analysis", European Journal of Communication, ${ }^{\circ} 1,1980$, pp. 151-171.

KATZ, Elihu et LIEBES, Tamar, "Once upon a time in Dallas", Intermedia, vol. 12, $n^{\circ} 3$, mai 1984 , pp. $28-32$.

KATZ Elihu, LreBES Tamar, "Decoding Dallas : Notes from a Cross-cultural Study", in GUMPERT and CATHCART (ed), Intermedia. New York, Oxford University Press, 1984, (repris dans NEWCOMB, H. (ed.) Television, the critical view, $4^{\mathrm{e}}$ ed., Oxford University Press, 1987, pp. 419-432).

KATZ, Elihu et LIEBES, Tamar, "Cross-cultural readings of Dallas : poetic and referential statements", Paper for the International Television studies conference, July 1984.

KATZ, Elihu et LuEBES, Tamar, "Mutual aid in the decoding of Dallas : preliminary notes from a cross-cultural study", in Philip DRUMMOND and Richard PATERSON (ed.), Television in transition, (Papers from the first international television studies conference), British Film Institute publishing, 1985, pp. 187-198.

KATZ, Elihu et LIEBES, Tamar, "Dallas and genesis : primordiality and seriality in television fiction", in James CAREY (ed.), Media, myths and narratives, Sage, 1987, pp. 113-125.

KATZ Elihu, LIEBES, Tamar, "Interacting with Dallas : cross cultural readings of american TV", Canadian Journal of Communication, vol. $15, \mathrm{n}^{\circ} 1$, hiver 1990, pp. 45-66.

KATZ Elihu, LIEBES Tamar, "Neither here nor there : japanese readings of Dallas", Communications, vol. 12, $\mathrm{n}^{\circ} 2$, juin 1991, pp. 99-110.

KaTz, Elihu et Liebes, Tamar, Six interprétations de la série Dallas, Hermès, $\mathrm{n}^{\circ} 11$ 12,1992 , pp. $125-144$. 
Kreutzner Gabriele, SeIter Ellen, "Not All 'Soaps' Are Created Equal : Towards a Crosscultural Criticism of Television Serials", Screen, Eynsham, Oxford, England, vol. 32, nº 2, été 1991, pp. 154-72.

LANCE Keith Curry, "From Buckingham Palace to Southfork Ranch : The Case for a Structuralist View of Popular Culture", Sociological-Spectrum, vol. 3, $\mathrm{n}^{\circ} 1$, Jan-Mar 1983, pp. 55-68.

LIEBES Tamar, "Ethnocentricism: Israelis of Moroccan ethnicity negotiate the meanings of Dallas", Studies in Visual Communications, vol. 10, n 3,1984 , pp. 46-72.

LIVINGSTONE Sonia, "The implicit representation of characters in Dallas : a multdimensional scaling approach", Human communication research, vol. 13, $\mathrm{n}^{\circ}$ 3, printemps 1987 , pp. 399-440.

MANDER, Mary. S., "Dallas: The mythology of crime and the occult, Journal of popular culture, vol. $17, \mathrm{n}^{\circ} 2,1983$, pp. 44-50.

MARTIN, Michèle, "Le téléroman : un produit culturel international", in Communication et médias de masse, (Culture, domination et opposition), Presses de l'université du Québec/ Télé-université, 1990, pp. 224-234.

MORIN Violette, "Le présent actif dans le feuilleton télévisé", Communications, Paris, $\mathrm{n}^{\circ} 39,1984$, pp. $239-246$.

NEWCOMB Horace, "Texas : a giant state of mind", Channels, vol. 1, $\mathrm{n}^{\circ} 1$, avril-mai 1981, repris dans NeWCOMB, H. (ed.), Television, the critical view, $4^{\circ}$ ed., Oxford University Press, 1987, pp. 419-432.

NIR, Yeshayahu, "J.R. , the Kennedys and Arik Sharon", Hotam, Summer 1984.

PORTNOY, Ethel, "Tramlijn begeerte", in Madonna's appel, Meulenhoff, 1990.

PUNTER David, The hidden script : writing and the unconscious, Institutional Affiliation Of First Author, in U East Anglia, Norwich NR4 7IJ England, Publisher Information Of Original Document (PB) : ix+193 pp., CI, London, England, Routledge \& Kegan Paul, 1985.

RAINTREe, Lee, Dallas, Paris, Robert Laffont, 1981.

RusHING, Janice-Hocker, "The Rhetoric of the American Western Myth", Communication-Monographs, Annandale, vol. 50, $\mathrm{n}^{\circ} 1$, mars 1983, pp. 1432.

SiLJ, Alessandro, A Est di Dallas : telefilm usa ed europei a confronto, Rome, RAI, 1988.

Sobell, Linda-C., SOBELl, Mark-B, RILEy Diane-M:, KlaINER Felix et al, "Effect of television programming and advertising on alcohol consumption in normal drinkers", Journal-of-Studies-on-Alcohol (Toronto), vol. 47, $\mathrm{n}^{\circ} 4,1986$, pp. 333-340.

Srouz, Joëlle, "Les Algériens regardent Dallas", in Les nouvelles chaînes, Paris/Genève, Presses Universitaires de France/Institut universitaire d'études du développement, 1983, pp. 223-246.

SWANSON Gillian, "Dallas", Framework, n' 14,1981 , pp. $32-35 ; n^{\circ} 15,1982$, p. 81 85.

TAN Alexis S., Lr Sarrina, SIMPSON Charles, "American TV and Social Stereotypes of Americans in Taiwan and Mexico", Journalism Quarterly, vol. 63, $\mathrm{n}^{\circ} 4$, hiver 1986, pp. 809-814.

Thomas, Sari et Callahan, Brian, P., "Allocating happiness : TV families and social class", Journal of communication, $\mathrm{n}^{\circ} 32 / 3,1982$, pp. 184-190.

Thoveron, Gabriel, “Dallas, hélàs! Mais Dinasty, nenni”, La Revue Nouvelle, 1983, pp. 17-25.

TYTell, Pamela, "De la Dallassomanie à la Dallassothérapie", Psychologie, $\mathrm{n}^{\circ} 157$, avril 1983, pp. 21-24. 
Wolf A. Sanford, "From Lassie to Dallas : A Critical View of the Hollywood TV Series", in SANTRAUD, Jeanne-Marie (ed.), Le Sud et autres points cardinaux, Actes du Colloque de 1984, Paris, Centre de Recherches en Lit. \& Civilisation N.-Amér., P. U. de Paris-Sorbonne, 1985, pp. 101-111.

\section{Presse et magazines}

El Moudjahid, ler octobre 1981.

Le Figaro, 31 mai 1982, DELAPRÉE Catherine, "C'est elle qui a tiré sur J.R.".

Le Monde, 10-11 novembre 1982, STOLZ, Joëlle, "L'Algérie regarde Dallas".

Le Monde diplomatique, $\mathrm{n}^{\circ} 349$, avril 1983, p. 26, RAMONET, Ignacio, ZIMMER, Christian, Tesson, Charles, "La télévision des temps durs", «Dallas»".

Le Nouvel Observateur, 15 mai 1982, pp. 78-79, SCHIFFRE, Alain, "Dallas :flagrant de Delly" ; 2 août 1985, pp. 46-47, ChOUFFAN, Alain, "Dallas : beau comme un feuilleton".

Le Point, $\mathrm{n}^{\circ}$ 507, 7 juin 1982, pp. 120-121, SUfferT, Georges, “Dallas : ah, les beaux salauds!"

Libération, 18-19 septembre 1982, pp. 18-22, LEFORT, G., RoussEAU, F., "Dallas, ça recommence et c'est déjà fini".

Newsweek, 17 novembre 1980, pp. 66-75, WATERs Harry F. et al, "Who shot that nice Mr. Ewing ?"

Skrien, $\mathrm{n}^{\circ} 118$, mai-juin 1982, TEE E., "Dallas : het gezin van de week".

The Gazette, 20 sept. 1986, JENNINGS, Diane, "J.R. Hated by all, except Larry Hagman".

Time Magazine, 11 août 1980, pp. 44-50, CoRLIss, Richard, “TV's Dallas : Whodunit ?".

TV Guide, vol. 34, 24 mai, 1986, pp. 34-44, LEAHY, Michael, "How Larry Hagman forced some changes in Dallas".

Washington Post, 12 dec. 1981, THORTON, Lesley.

\section{Audio-visuel}

Faire Dallas, réal. José Varela, INA, TF1, 1984.

Télé-dollars, (Une enquête dans les coulisses de la télévision) de Max Cacopardo, première partie : "Rose-Anna vs J.R", Société Radio-Canada, 1987.

L'envers de Dallas, "Nouveau vendredi", FR3, 1er oct. 1982. 